\title{
Evaluation of laparoscopic surgery for small bowel obstruction and factors related to outcomes
}

\author{
Hajime Kayano ${ }^{1}$, Eiji Nomura ${ }^{1}$, Yasuhiko Ueda ${ }^{1}$, Toru Kuramoto ${ }^{2}$, Takashi Machida $^{1}$, Shuji Uda $^{1}$, Masaya Mukai $^{1}$, \\ Seiichiro Yamamoto ${ }^{3}$, Hiroyasu Makuuchi ${ }^{1}$ \\ ${ }^{1}$ Department of General and Gastroenterological Surgery, Tokai University Hachioji Hospital, Tokyo, Japan \\ ${ }^{2}$ Department of General and Gastroenterological Surgery, Saiseikai Suita Hospital, Osaka, Japan \\ ${ }^{3}$ Department of Surgery, Tokai University School of Medicine, Kanagawa, Japan
}

Videosurgery Miniinv 2020; 15 (2): 268-275

DOI: https://doi.org/10.5114/wiitm.2020.93243

\begin{abstract}
Introduction: In recent years, laparoscopic surgery (LS) has been performed for small bowel obstruction (SBO). However, the indications and short-term and long-term outcomes of LS for SBO have not yet been established.

Aim: To evaluate the usefulness of LS for SBO compared to open surgery (OS), as well as to identify risk factors for poor outcomes after $L S$.

Material and methods: A total of 105 patients who underwent surgery for SBO were divided into OS $(n=64)$ and $L S(n=41)$ groups, and propensity score-matched analysis was used to compare the short-term and long-term outcomes of the groups. Risk factors for conversion to OS, postoperative complications, and intraoperative bowel injury in LS were also identified.

Results: The incidences of surgical site infection and postoperative ileus were significantly lower in the LS group. The incidence of recurrent bowel did not differ significantly between the two groups. Prior bowel obstruction was a risk factor for conversion of $L S$ to OS (odds ratio $(O R)=24.79, p=0.0025)$. Bowel diameter was a risk factor for postoperative complications $(O R=1.50,95 \% \mathrm{Cl}: 1.01-2.22)$ and for bowel injury $(O R=1.33,95 \% \mathrm{Cl}: 1.05-1.67)$.

Conclusions: $L S$ for SBO had better postoperative short-term outcomes than OS. The outcomes of LS for SBO were significantly affected by prior bowel obstruction and bowel diameter.
\end{abstract}

Key words: small bowel obstruction, laparoscopic surgery, outcome, risk factors.

\section{Introduction}

Methods of treatment of small bowel obstruction (SBO) vary from patient to patient, and some respond well to decompression and other conservative therapies. Unfortunately, there are not a few cases that have ultimately required surgical treatment [13]. Open surgery (OS) has conventionally been the first-choice surgical treatment because of the risk of bowel injury and surrounding organs due to severe intraperitoneal adhesions. Recent advances in laparoscopic techniques [4, 5] and instruments [6] have enabled the development of standardized procedures for all types of abdominal diseases. As a result, laparoscopic surgery (LS) has gradually changed from being used as a diagnostic method for SBO to being used as a therapeutic method, and it is now expected to be used as a minimally invasive therapeutic technique that has been performed in many institutions. The advantages of LS, such as shortening of postoperative hospitalization and reduction of postoperative complications, have been reported

\section{Address for correspondence}

Hajime Kayano MD, PhD, Department of General and Gastroenterological Surgery, Tokai University Hachioji Hospital,

1838 Ishikawa-machi, 192-0032, Hachioji, Tokyo, Japan, phone: +81 426391111, e-mail: h.kayano@tsc.u-tokai.ac.jp 
$[7,8]$. However, LS for SBO is technically more difficult than OS, and it is more difficult to develop the operative field and use forceps than in LS for other abdominal diseases, and problems related to conversion to OS and intraoperative bowel injury due to these problems have been reported $[9,10]$. Therefore, no consensus on its outcomes and indications has yet been reached [11-14]. In this study, the usefulness of LS for SBO was evaluated by comparing its outcomes with those of conventional OS, and risk factors associated with negative outcomes such as conversion to OS, postoperative complications, and intraoperative bowel injury in LS were evaluated to overcome the problems of LS for SBO.

\section{Aim}

The aim of this study was to evaluate the impact of LS on postoperative complications and postoperative ileus, as well as to identify risk factors for conversion, postoperative complications, and bowel injury in the LS group.

\section{Material and methods}

\section{Study design}

The subjects of this study were 105 patients who underwent either LS (41 patients) or OS (64 patients) between October 2012 and June 2018 for SBO, excluding cases diagnosed as having SBO due to malignant diseases such as peritoneal metastasis after radical surgery for abdominal malignant tumor, small intestinal cancer, and malignant lymphoma by definitive diagnosis after surgery, and SBO due to an incarcerated inguinal or obturator/femoral/ any abdominal hernia. Patient attributes, operative time, blood loss, conversion to OS, intraoperative and postoperative complications, date of first passage of gas, date of resumption of eating, and duration of hospital stay were recorded in a database. All patients underwent OS or LS after having received a sufficient explanation of each method and its risks, and they provided their informed consent. The study protocol was approved by the Institutional Review Board for Clinical Research, Tokai University (18R-197) and was performed in accordance with its guidelines and regulations. All patients provided written, informed consent for the use of their clinical data. The primary outcomes of the study were postoperative complications and postoperative ileus.
Postoperative complications were divided according to the time of their occurrence as short-term if they occurred between the completion of surgery and initial postoperative discharge or long-term if they occurred after initial postoperative discharge. The two groups were matched by propensity scores, and their postoperative short-term and long-term outcomes were compared. Postoperative complications were defined as those of Clavien-Dindo classification (CD) grade II or higher [15]. Postoperative ileus and recurrent bowel obstruction were defined as requiring fasting, intravenous fluids, and decompression, and they were diagnosed by abdominal signs and abdominal computed tomography (CT), plain radiography, and ultrasound. Intraoperative bowel injury was defined as cases of full-thickness bowel injury requiring small bowel resection and anastomosis, and cases where serosal muscular suture was performed only for serosal muscular layer bowel injury. Conversion to OS was defined as the use of an additional skin incision measuring $\geq 5 \mathrm{~cm}$. Risk factors for conversion to OS, postoperative complications, and intraoperative bowel injury were also analyzed for all patients in the LS group. The factors investigated as potential risk factors for conversion to OS and intraoperative bowel injury during LS were sex, age, American Society of Anesthesiologists Physical Status Classification (ASA-PS) $\geq 3$, body mass index $(B M I) \geq 25 \mathrm{~kg} / \mathrm{m}^{2}$, number of prior operations, prior OS, prior bowel obstruction, maximum dilated small bowel diameter on preoperative abdominal $\mathrm{CT}$, cause of SBO (adhesion, isolated band, torsion, or internal hernia) based on intraoperative findings, and longtube placement. The factors investigated as potential risk factors for postoperative complications were those listed above with the addition of conversion to OS and intraoperative bowel injury in all LS cases.

\section{Preoperative management and surgical procedure}

LS was indicated for all patients with SBO other than those with extensive intestinal necrosis and those with unstable respiratory and circulatory dynamics, and since 2017, it has been regarded as the first-choice treatment. Whenever possible, a long tube was placed, intestinal decompression was performed before surgery, and the stenotic area was evaluated using a water-soluble gastrointestinal contrast agent. In the surgical procedure, the umbi- 
licus was the first-choice insertion site for the first 12-mm trocar, but if preoperative abdominal contrast-enhanced $\mathrm{CT}$ or ultrasound suggested that severe adhesions might be present at this site, the left or right abdomen was used instead, and the open method was selected. Pneumoperitoneum was induced, and the severity of intraperitoneal adhesions was assessed, after which $5-\mathrm{mm}$ trocars were inserted on the basis of forming a triangle with the culprit lesion at the center. When extending the operation field was difficult, further 5 - $\mathrm{mm}$ trocars were added as necessary. If small bowel resection was required, the $12-\mathrm{mm}$ trocar insertion wound was extended to perform mini-open surgery, through which the small bowel was brought outside the body for resection and anastomosis.

\section{Statistical analysis}

Comparisons between the two groups before propensity score (PS) matching were performed using the Mann-Whitney test to compare continuous variables and Pearson's $\chi^{2}$ test to compare categorical variables. For PS matching, preoperative variables in the LS and OS groups were first compared by univariate analysis, after which multiple logistic regression analysis was performed using only those factors identified as significant. These factors were used to calculate propensity scores, and the LS and OS groups were then matched $1: 1$ using a caliper set at 0.2. Comparisons between the two groups after PS matching were performed using the Wilcoxon signed-rank test to compare continuous variables and McNemar's test to compare categorical variables. Risk factors were identified by the stepwise backward selection method. JMP software for Windows, version 13.0 (SAS Institute, Cary, NC, USA) was used for all statistical analyses, and $p<0.05$ was regarded as significant.

\section{Results}

\section{Patient characteristics of the baseline and propensity-matched cohorts}

Table I shows a comparison of the attributes of all patients in the OS and LS groups. The LS group contained significantly fewer patients with ASA-PS $\geq 3$ and patients with prior open surgery compared with the OS group. The rate of long-tube placement was also significantly higher in the LS group, and internal hernia and isolated band were also significantly more common causes of SBO. Multivariate logistic analysis using ASA-PS $\geq 3$, prior open surgery, long-tube placement, and internal hernia or isolated band as the cause of SBO as covariates identified prior open surgery (odds ratio $(O R)=0.20$, 95\% confidence interval (Cl): 0.06-0.67), long-tube placement $(\mathrm{OR}=5.19,95 \% \mathrm{Cl}: 1.61-16.65)$, and internal hernia $(\mathrm{OR}=14.25,95 \% \mathrm{Cl}: 2.29-88.65)$ as significant factors (Table II). After propensity score matching, 26 matched pairs were selected (Table I). The standardized difference score after propensity matching was -0.07 for prior open surgery, -0.07 for long-tube placement, and 0.09 for internal hernia (Table II). The area under the curve (AUC) of the receiver operating characteristic (ROC) curve was 0.79, indicating moderate accuracy.

\section{Short-term outcomes in the matched cohort}

Table III shows a comparison of the short-term outcomes for the two groups. Operative time was longer for LS than for OS, but no significant difference was observed. Blood loss was significantly lower ( $25 \mathrm{ml}$ vs. $77.5 \mathrm{ml}, p=0.0293$ ), and postoperative hospital stay was significantly shorter (10.5 days vs. 14 days, $p=0.0245$ ) in the LS group than in the OS group. The incidence of postoperative complications was also significantly lower in the LS group (15.3\% vs. $42.3 \%, p=0.0297)$. In terms of postoperative complications, the incidences of SSI (3.8\% vs. $42.3 \%, p=0.0153)$ and postoperative ileus ( $\%$ vs. $11.5 \%, p=0.0372$ ) were significantly lower in the LS group. Bowel injuries tended to be more common in the LS group than in the OS group $(30.7 \%$ vs. $19.2 \%$, $p=0.2941)$, but the difference was not significant.

\section{Long-term outcomes in the matched cohort}

The only long-term postoperative complication evaluated was the incidence of recurrent bowel obstruction, and this did not differ significantly between the two groups (Table III). There was no significant difference between the two groups in the observation period.

\section{Details of surgical outcomes of LS for SBO}

For all cases of $\mathrm{LS}$, the conversion rate to OS was $21.9 \%$. The reason for conversion to OS was 
Table I. Patient characteristics

\begin{tabular}{|c|c|c|c|c|c|c|}
\hline \multirow[t]{2}{*}{ Parameter } & \multicolumn{3}{|c|}{ Unmatched } & \multicolumn{3}{|c|}{ PS-matched } \\
\hline & $\begin{array}{l}\text { Laparoscopic } \\
\quad(n=41)\end{array}$ & $\begin{array}{l}\text { Open } \\
(n=64)\end{array}$ & $P$-value & $\begin{array}{l}\text { Laparoscopic } \\
\quad(n=26)\end{array}$ & $\begin{array}{l}\text { Open } \\
(n=26)\end{array}$ & $P$-value \\
\hline Sex (male) (\%) & $28(68.2)$ & $37(57.8)$ & 0.2807 & $16(61.5)$ & $16(61.5)$ & 1.0000 \\
\hline Age [years] ${ }^{a}$ & $71(24-89)$ & $72(26-97)$ & 0.2000 & $73(34-89)$ & $69(26-85)$ & 0.2338 \\
\hline ASA-PS $\geq 3$ (\%) & $12(29.2)$ & $36(56.2)$ & $0.0068^{*}$ & $12(46.1)$ & $13(50.0)$ & 0.7814 \\
\hline $\mathrm{BMI} \geq 25 \mathrm{~kg} / \mathrm{m}^{2}(\%)$ & $2(4.8)$ & $4(6.2)$ & 0.7676 & $2(7.6)$ & $1(3.8)$ & 0.552 \\
\hline Prior surgery $\geq 2$ (\%) & $12(29.2)$ & $22(34.3)$ & 0.5854 & $11(42.3)$ & $8(30.7)$ & 0.3876 \\
\hline $\begin{array}{l}\text { Prior open surgery } \\
\text { (\%) }\end{array}$ & $21(51.2)$ & $53(82.8)$ & $0.0005^{*}$ & $20(76.9)$ & $19(73.0)$ & 0.7488 \\
\hline $\begin{array}{l}\text { Prior laparoscopic } \\
\text { surgery (\%) }\end{array}$ & $6(14.6)$ & $4(6.2)$ & 0.1533 & $1(3.8)$ & $3(11.5)$ & 0.2980 \\
\hline $\begin{array}{l}\text { CT maximum bowel } \\
\text { diameter }[\mathrm{mm}]^{a}\end{array}$ & $36.7(21.4-53.3)$ & $35.85(20-69.2)$ & 0.6110 & $36.75(21.5-53.3)$ & $35.6(20.0-51.5)$ & 0.7734 \\
\hline $\begin{array}{l}\text { Prior bowel } \\
\text { obstruction (\%) }\end{array}$ & $5(12.2)$ & 10 (15.9) & 0.6241 & $5(19.2)$ & $5(19.2)$ & 1.0000 \\
\hline $\begin{array}{l}\text { Long-tube } \\
\text { placement (\%) }\end{array}$ & $27(65.8)$ & $28(43.7)$ & $0.0269^{\star}$ & $16(61.5)$ & $17(65.3)$ & 0.7734 \\
\hline \multicolumn{7}{|l|}{ Etiology of SBO: } \\
\hline Adhesion (\%) & $15(36.5)$ & $23(35.9)$ & 0.9463 & $13(50.0)$ & $13(50.0)$ & 1.0000 \\
\hline Isolated band (\%) & $32(50.0)$ & $10(24.3)$ & $0.0090^{*}$ & $9(34.6)$ & $9(34.6)$ & 1.0000 \\
\hline Torsion (\%) & $2(4.8)$ & $7(10.9)$ & 0.2792 & $1(3.8)$ & $2(7.6)$ & 0.5520 \\
\hline Internal hernia (\%) & $14(34.1)$ & $2(3.1)$ & $0.0001^{*}$ & $3(11.5)$ & $2(7.6)$ & 0.6381 \\
\hline $\begin{array}{l}\text { White blood cell } \\
\text { count }\left[\mathrm{mm}^{3}\right]^{a}\end{array}$ & $\begin{array}{c}6500 \\
(3200-14200)\end{array}$ & $\begin{array}{c}8300 \\
(2500-29500)\end{array}$ & 0.0992 & $\begin{array}{c}6300 \\
(3200-14200)\end{array}$ & $\begin{array}{c}7850 \\
(2900-29500)\end{array}$ & 0.4640 \\
\hline $\begin{array}{l}\text { C-reactive protein } \\
{[\mathrm{mg} / \mathrm{dl}]^{a}}\end{array}$ & $\begin{array}{c}0.47 \\
(0.01-24.51)\end{array}$ & $\begin{array}{c}0.52 \\
(0.01-35.50)\end{array}$ & 0.7032 & $\begin{array}{c}0.62 \\
(0.01-24.54)\end{array}$ & $\begin{array}{c}0.52 \\
(0.01-19.23)\end{array}$ & 0.9489 \\
\hline
\end{tabular}

PS - propensity score, ASA-PS - American Society of Anesthesiologists physical status classification, BMI - body mass index, CT - computed tomography, $S B O-$ small bowel obstruction, a Values shown as medians (range), ${ }^{*} p<0.05$.

Table II. Factors affecting surgical method selection

\begin{tabular}{|lcccc|}
\hline Variable & Odds ratio & $95 \% \mathrm{Cl}$ & $P$-value & Std diff score \\
\hline ASA-PS $\geq 3$ & 0.55 & $0.21-1.45$ & 0.2329 & -0.07 \\
\hline Prior open surgery & 0.20 & $0.06-0.67$ & $0.0092^{*}$ & 0.08 \\
\hline Long-tube placement & 5.19 & $1.61-16.65$ & $0.0056^{*}$ & -0.07 \\
\hline Isolated band & 1.30 & $0.43-3.92$ & 0.6351 & 0.00 \\
\hline Internal hernia & 14.25 & $2.29-88.65$ & $0.0001^{*}$ & 0.09 \\
\hline
\end{tabular}

Cl - confidence interval, Std diff - standardized difference. ${ }^{*} \mathrm{P}<0.05$, A standardized difference score (Std diff score) of $<0.1$ suggests adequate variable balance after propensity matching. 
Table III. Comparison of outcomes after propensity score matching

\begin{tabular}{|c|c|c|c|}
\hline \multirow[t]{2}{*}{ Variable } & \multicolumn{3}{|c|}{ PS-matched } \\
\hline & Laparoscopic $(n=26)$ & Open $(n=26)$ & $P$-value \\
\hline \multicolumn{4}{|l|}{ Short-term outcomes: } \\
\hline Operative time $[\mathrm{min}]^{a}$ & $121(46-252)$ & $98(30-288)$ & 0.0558 \\
\hline Blood loss $[\mathrm{ml}]^{\mathrm{a}}$ & $25(2-480)$ & $77.5(5-1960)$ & $0.0293^{*}$ \\
\hline Conversion (\%) & $6(23.0)$ & & \\
\hline Bowel injury (\%) & $8(30.7)$ & $5(19.2)$ & 0.2941 \\
\hline Time to first bowel movement [days] & $2(1-6)$ & $3(1-6)$ & 0.0977 \\
\hline Time to resumption of soft diet [days] ${ }^{a}$ & $5(1-21)$ & $5(2-22)$ & 0.5216 \\
\hline Count of analgesic use [times] ${ }^{a}$ & $1.5(0-23)$ & $2.5(0-39)$ & 0.1590 \\
\hline Postoperative hospital stay [days] ${ }^{a}$ & $10.5(4-90)$ & $14(7-60)$ & $0.0245^{*}$ \\
\hline Overall complications (\%) & $4(15.3)$ & $11(42.3)$ & $0.0297^{*}$ \\
\hline SSI (\%) & $1(3.8)$ & $7(26.9)$ & $0.0153^{*}$ \\
\hline Aspiration pneumonia (\%) & $1(3.8)$ & $1(3.8)$ & 1.0000 \\
\hline Anastomotic leakage (\%) & $1(3.8)$ & $0(0)$ & 0.2357 \\
\hline Port site hernia (\%) & $1(3.8)$ & $0(0)$ & 0.2357 \\
\hline Postoperative ileus (\%) & $0(0)$ & $3(11.5)$ & $0.0372^{*}$ \\
\hline \multicolumn{4}{|l|}{ Long-term outcomes: } \\
\hline Recurrence (\%) & $0(0)$ & $1(3.8)$ & 0.2357 \\
\hline Observation period [days] $]^{a}$ & $127(16-1300)$ & 315.5 (9-1779) & 0.1053 \\
\hline
\end{tabular}

SSI - surgical site infection, ${ }^{a}$ Values shown as medians (range), ${ }^{*} p<0.05$.

severe adhesions in 4 cases, difficulty securing the operative field in 3 cases, and wide-range necrosis in 2 cases. The intraoperative bowel injury rate was $21.9 \%$. Full-thickness bowel injury was seen in 5 cases, and serosal muscular layer bowel injury was seen in 4 cases. The overall complication rate was $14.6 \%$ (Table IV).

\section{Risk factors for conversion, postoperative complications, and bowel injury}

Prior bowel obstruction was a risk factor for conversion to $\mathrm{OS}(\mathrm{OR}=24.79,95 \% \mathrm{Cl}$ : 2.28-131.1). Bowel diameter was a risk factor for postoperative complications (OR $=1.50,95 \% \mathrm{Cl}: 1.01-2.22)$ and for bowel injury $(\mathrm{OR}=1.33,95 \% \mathrm{Cl}$ : 1.05-1.67) (Table V).

\section{Discussion}

LS for abdominal disease has now been standardized, like cholecystectomy, appendectomy, col- ectomy, and gastrectomy, etc., with advances in laparoscopic techniques and instruments. Reports of the use of LS for SBO, for which OS has conventionally been the first-choice treatment, have also been increasing in recent years. LS was initially used as a diagnostic tool in the treatment of SBO [16], but today it is also used as a method of treatment. However, there is as yet no consensus on the indications for LS for SBO or on its short-term and long-term postoperative outcomes [11-14]. In the present study, length of hospital stay was shorter, and the incidence of complications was lower for LS than for OS, and postoperative ileus and SSI were significantly decreased in LS. These results suggest that LS may be superior to open surgery in terms of short-term postoperative outcomes, and that its advantages as a minimally invasive procedure may also be applicable to SBO surgery. Some studies have found that LS for SBO may reduce the rates of postoperative ileus compared with OS [17]. In 
Table IV. Details of surgical outcomes of LS for SBO

\begin{tabular}{|lc|}
\hline Parameter & Total $(n=41)$ \\
\hline Operative time [min] $^{a}$ & $120(38-311)$ \\
\hline Blood loss [ml] $^{a}$ & $7(2-480)$ \\
\hline Conversion (\%) & $9(21.9)$ \\
\hline Severe adhesion & 4 \\
\hline Difficulty securing operative field & 3 \\
\hline Wide-range necrosis & 2 \\
\hline Intraoperative bowel injury (\%) & $9(21.9)$ \\
\hline Full-thickness bowel injury & 5 \\
\hline Serosal muscular layer bowel injury & 4 \\
\hline Overall complication (\%) & $6(14.6)$ \\
\hline SSI & 2 \\
\hline Anastomotic leakage & 1 \\
\hline Abscess & 1 \\
\hline Port site hernia & 1 \\
\hline Aspiration pneumonia & 1 \\
\hline${ }^{a}$ Values shown as medians (range) & \\
\hline
\end{tabular}

the present study as well, LS helped prevent the short-term complication of postoperative ileus. On the other hand, in the present study, LS was equivalent to OS in recurrence of SBO, as reported so far, and it could not be demonstrated that it was superior. Major issues with the use of LS for SBO are conversion to OS and intraoperative bowel injury. The conversion rate of LS for SBO has been reported to be $29-33.6 \%[7,18]$ so far and was $21 \%$ in the present study. Prior studies have found that factors increasing the risk of conversion to OS include bowel diameter $\geq 4 \mathrm{~cm}$ [19] and three or more prior open abdominal procedures [20], but the only risk factor identified in the present study was prior bowel obstruction (1.6 times). Possible reasons for conversion to OS are severe adhesions and difficulty extending the operative field. Many patients with prior bowel obstruction have severe or multiple adhesions, making it difficult to observe and grasp the course of the bowel laparoscopically, and this was a common reason for conversion. In such patients, preoperative intestinal decompression may also have been poor, leaving the bowel markedly dilated and making it difficult to secure the operative
Table V. Multivariate analysis of risk factors for conversion, postoperative complications, and intraoperative bowel injury

\begin{tabular}{|c|c|c|c|}
\hline Risk factors & $\begin{array}{l}\text { Odds } \\
\text { ratio }\end{array}$ & $95 \% \mathrm{Cl}$ & $P$-value \\
\hline \multicolumn{4}{|l|}{ Conversion risk factors: } \\
\hline Prior bowel obstruction & 24.79 & $2.28-269.60$ & $0.0025^{\star}$ \\
\hline \multicolumn{4}{|c|}{ Risk factors for postoperative complications: } \\
\hline Bowel diameter & 1.50 & $1.01-2.22$ & $0.0001^{*}$ \\
\hline \multicolumn{4}{|c|}{ Risk factors for intraoperative bowel injury: } \\
\hline Bowel diameter & 1.33 & $1.05-1.67$ & $0.0001^{*}$ \\
\hline
\end{tabular}

field, and this may also be a risk factor for conversion to OS. To overcome these disadvantages of LS, we carry out preoperative long-tube placement as much as possible in both emergency and elective procedures. The long tube is reportedly superior to the gastric tube in the treatment of SBO [21-23]. In the case of SBO in which the presence of intestinal necrosis has been ruled out, after long-tube insertion, we start by providing medical treatment and assess its effectiveness on day 7 after its placement. If it has been ineffective, the long tube is left in place until surgery, and intestinal decompression is performed as much as possible. The advantage of long-tube placement is that, because it is more difficult to explore the entire bowel during LS than during OS, preoperative contrast enhancement can be performed to identify the responsible lesion, and a search for the responsible lesion can also be performed intraoperatively using the long tube as a guide, reducing the burden during LS. Small bowel diameter is a risk factor for postoperative complications, and although long-tube placement was not found to have had any effect in the present study, preoperative intestinal decompression to reduce the diameter of the small bowel and confirm its course by preoperative contrast enhancement may help reduce the incidence of postoperative complications. Bowel injury is reportedly more common in LS than in OS [9]. In some cases, not only must serosal muscular layer bowel injury be repaired, but bowel resection may be required when the intestinal tract is extensively damaged across all layers. In the previous reports $[9,10,17,18]$, the incidence rate was high, at $4.8-37 \%$. In the present study, there was 
no significant difference in the incidence of bowel injury, but the rate did tend to be higher for LS, and small bowel diameter was a risk factor for bowel injury. Compared with OS, bowel dilation does make it more difficult to secure a space for manipulation in LS, and accidental bowel injury is more likely to occur. Intestinal decompression is the best way to prevent these problems, and a long tube should be placed. In addition, there have been reports of delayed intestinal damage in LS, and greater caution is required in LS than in OS. In order to avoid intestinal damage, we use bipolar scissors (AESCULAP AdTec single use bipolar B.BRAUN, Melsungen, Germany) when removing intestinal adhesions.

Another important issue with LS for SBO is its greater technical difficulty compared with OS. Differences in surgeons' familiarity with LS for SBO have a major effect on surgical outcomes [24], and it has been claimed that this technique should only be used by surgeons who are proficient in it $[10,25]$. Studies have identified a learning curve in skill acquisition in LS [26, 27]. To shorten this learning curve, surgical procedures must be standardized. However, LS for $\mathrm{SBO}$ is difficult to standardize, and before performing this procedure, surgeons must therefore become proficient in the standardized laparoscopic surgical procedures for other disorders, such as those of the stomach and colon; in particular, they should learn how to handle the bowel with forceps and how to extend the operative field.

The limitations of this study are its retrospective, non-randomized, and single-center design. In addition, despite the considerable sample size of the entire study population, it might still be too small for adequate subgroup analyses. Despite all these limitations, this study strongly suggests that LS has some advantages for SBO. Data from prospective, randomized, controlled trials of LS for this indication are needed.

\section{Conclusions}

LS is a useful approach for SBO, and preoperative decompression, patient selection, and the gradual expansion of indications may enable more patients to benefit from the advantages of this minimally invasive treatment.

\section{Conflict of interest}

The authors declare no conflict of interest.

\section{References}

1. Seror D, Feigin E, Szold A, et al. How conservatively can postoperative small bowel obstruction be treated? Am J Surg 1993; 165: $121-5$.

2. Tanaka S, Yamamoto T, Kubota D, et al. Predictive factors for surgical indication in adhesive small bowel obstruction. Am J Surg 2008; 196: 23-7.

3. Fevang BT, Jensen D, Fevang J, et al. Upper gastrointestinal contrast study in the management of small bowel obstruction: a prospective randomised study. Eur J Surg 2000; 166: 39-43.

4. Lee SW, Kawai M, Tashiro K, et al. The crossover technique for intracorporeal esophagojejunostomy following laparoscopic total gastrectomy: a simple and safe technique using a linear stapler and two barbed sutures. Surg Endosc 2019; 33: 1386-93.

5. Kwiatkowski AP, Stępińska G, Stanowski E, et al. Intracorporeal versus extracorporeal anastomosis in laparoscopic right hemicolectomy - single center experience. Videosurgery Miniinv 2019; 14: 381-6.

6. Yamamoto S, Kanai T, Osumi K, et al. Anastomotic leakage using linear stapling device with pre-attached bioabsorbable polyglycolic acid felt after laparoscopic anterior resection. Anticancer Res 2017; 37: 7083-6.

7. Lombardo S, Baum K, Filho JD, et al. Should adhesive small bowel obstruction be managed laparoscopically? A National Surgical Quality Improvement Program propensity score analysis. J Trauma Acute Care Surg 2014; 76: 696-703.

8. Byrne J, Saleh F, Ambrosini L, et al. Laparoscopic versus open surgical management of adhesive small bowel obstruction: a comparison of outcomes. Surg Endosc 2015; 29: 2525-32.

9. Behman R, Nathens AB, Byrne JP, et al. Laparoscopic surgery for adhesive small bowel obstruction is associated with a higher risk of bowel injury: a population-based analysis of 8584 patients. Ann Surg 2017; 266: 489-98.

10. Di Saverio S, Birindelli A, Broek RT, et al. Laparoscopic adhesiolysis: not for all patients, not for all surgeons, not in all centres. Updates Surg 2018; 70: 557-61.

11. Yao S, Tanaka E, Matsui Y, et al. Does laparoscopic adhesiolysis decrease the risk of recurrent symptoms in small bowel obstruction? A propensity score-matched analysis. Surg Endosc 2017; 31: 5348-55.

12. Patel R, Borad NP, Merchant AM. Comparison of outcomes following laparoscopic and open treatment of emergent small bowel obstruction: an 11-year analysis of ACS NSQIP. Surg Endosc 2018; 32: 4900-11.

13. Sajid MS, Khawaja AH, Sains P, et al. A systematic review comparing laparoscopic vs open adhesiolysis in patients with adhesional small bowel obstruction. Am J Surg 2016; 212: 138-50.

14. Pei KY, Asuzu D, Davis KA. Will laparoscopic lysis of adhesions become the standard of care? Evaluating trends and outcomes in laparoscopic management of small-bowel obstruction using the American College of Surgeons National Surgical Quality Improvement Project Database. Surg Endosc 2017; 31: 2180-6.

15. Dindo D, Demartines N, Clavien PA. Classification of surgical complications: a new proposal with evaluation in a cohort of 6336 patients and results of a survey. Ann Surg 2004; 240: 205-13. 
16. Peterson-Brown S, Vipond MN. Modern aids to clinical decision making in the acute abdomen. Br J Surg 1990; 77: 13-8.

17. Nakamura T, Sato T, Naito M, et al. Laparoscopic surgery is useful for preventing recurrence of small bowel obstruction after surgery for postoperative small bowel obstruction. Surg Laparosc Endosc Percutan Tech 2016; 26: e1-4.

18. O'Connor DB, Winter DC. The role of laparoscopy in the management of acute small-bowel obstruction: a review of over 2,000 cases. Surg Endosc 2012; 26: 12-7.

19. Suter M, Zermatten P, Halkic N, et al. Laparoscopic management of mechanical small bowel obstruction: are there predic tors of success or failure? Surg Endosc 2000; 14: 478-83.

20. Farinella E, Cirocchi R, La Mura F, et al. Feasibility of laparoscopy for small bowel obstruction. World J Emerg Surg 2009; 4: 3.

21. Chen $\mathrm{XL}$, Ji F, Lin $\mathrm{Q}$, et al. A prospective randomized trial of transnasal ileus tube vs nasogastric tube for adhesive small bowel obstruction. World I Gastroenterol 2012; 18: 1968-74.

22. Li RH, Li DC, Lv XP, et al. Radiophotographically controlled nasointestinal intubation to treat recurrent postoperative adhesive ileus. Cell Biochem Biophys 2015; 72: 509-14.

23. Gowen GF. Long tube decompression is successful in $90 \%$ of patients with adhesive small bowel obstruction. Am J Surg 2003; 185: 512-5.

24. Oyasiji T, Helton SW. Survey of opinions on operative management of adhesive small bowel obstruction: laparoscopy versus laparotomy in the state of Connecticut. Surg Endosc 2011; 25: 2516-21.

25. Szeliga J, Jackowski M. Laparoscopy in small bowel obstruction current status - review. Videosurgery Miniinv 2017; 12: 455-60.

26. Kayano H, Okuda J, Tanaka K, et al. Evaluation of the learning curve in laparoscopic low anterior resection for rectal cancer. Surg Endosc 2011; 25: 2972-9.

27. Yamamoto M, Okuda J, Tanaka K, et al. Evaluating the learning curve associated with laparoscopic left hemicolectomy for colon cancer. Am Surg 2013; 79: 366-71.

Received: 8.01.2020, accepted: 20.01.2020. 